\title{
Output-Based Control of Robots with Variable Stiffness Actuation
}

\author{
Gianluca Palli and Claudio Melchiorri \\ Dipartimento di Elettronica, Informatica e Sistemistica, Università di Bologna, Viale Risorgimento 2, 40136 Bologna, Italy \\ Correspondence should be addressed to Gianluca Palli, gianluca.palli@unibo.it
}

Received 12 April 2011; Accepted 25 August 2011

Academic Editor: G. Muscato

Copyright ( 2011 G. Palli and C. Melchiorri. This is an open access article distributed under the Creative Commons Attribution License, which permits unrestricted use, distribution, and reproduction in any medium, provided the original work is properly cited.

\begin{abstract}
The output-based control of a redundant robotic manipulator with relevant and adjustable joint stiffness is addressed. The proposed controller is configured as a cascade system that allows the decoupling of the actuators dynamics from the arm dynamics and the consequent reduction of the order of the manipulator dynamic model. Moreover, the proposed controller does not require the knowledge of the whole robot state: only the positions of the actuators and of the joints are necessary. This approach represents a significant simplification with respect to previously proposed state feedback techniques. The problem of controlling simultaneously the position trajectory and the desired stiffness in both the joint and work space is investigated, and the relations between the manipulator redundancy and the selection of both the joint and work space stiffness of the manipulator are discussed. The effectiveness of the proposed approach is verified by simulations of a 3 degrees of freedom planar manipulator.
\end{abstract}

\section{Introduction}

Physical human-robot interaction currently represents one of the most challenging issues in robotics. To this end, a new class of robots is under development considering safety and dependability as a primary goal. While early applications of the variable stiffness concept were developed for prosthetic purposes due to the bio-inspired ability of these actuation systems of emulating the behavior of human muscles [1], variable stiffness actuators have been recently introduced in robotics to achieve a suitable tradeoff between accuracy in tasks execution and safety while operating in unstructured environments and in particular in the case of physical interaction/cooperation with humans. In [2], it has been shown how, due to the high reduction ratio of the gearboxes usually adopted in the transmission systems of robotic arms, the kinetic energy of the actuation system is predominant with respect to the one of the arm structure. Moreover, while the inertia of the robot arm can be reduced by adopting lightweight structures and composite materials, it is more difficult to reduce the inertia of the actuators and of the gearboxes because of technological limitations. Due to these considerations, Bicchi and Tonietti [2] proposed to adopt variable stiffness actuation to make the robot more rigid when the system velocities (and then kinetic energy) are low and to make the robot more compliant (or soft) during fast motions (that means high kinetic energy) so that the transmission system may store at least part of the kinetic energy of the actuation system in case unpredictable impacts occur. On the other hand, the kinetic energy of the robotic arm structure can be absorbed during impacts by suitable soft covers mounted on the robot [3], emulating in this way the function and the behavior of the human skin. The research effort in this field is motivated by the potential benefits given by the introduction of robots in human everyday activities, such as assistance to elder or handicapped people, homework activities, and entertainment. It is clear that purposely designed robots are needed to accomplish this kind of tasks.

Although several prototypes of single degree-of-freedom (DOF) joints with relevant and adjustable compliance for robotic manipulators have been designed and developed [48 ] with the aim of verifying the effectiveness of the variable stiffness approach, the implementation of a robotic arm with variable stiffness actuation is still an issue, and only recently, the German Aerospace Center (DLR) has started the development of such a robot. From the mechanical point of view, the main drawbacks of variable stiffness joints are a 
more complex mechanical design and the necessity of a pair of actuators to adjust simultaneously both the joint position and stiffness. It follows that a more complicated modeling and control approach is necessary, especially when kinematic structures with multiple DOF are considered [9]. In [10], a general approach to the feedback linearization and the simultaneous decoupled control of both the position and stiffness in a serial manipulator actuated by means of variable stiffness joints is proposed, while in [11], a robust controller for the compensation of the model uncertainties in variable stiffness robotic manipulators is presented.

In this paper, the control of robotic manipulators with variable joint stiffness is addressed in the general case of a redundant arm by means of a simplified (with respect to previous ones) output-feedback control approach. The proposed control is configured as a cascade system composed by three independent controllers, one for each part in which the dynamics of the robotic arm with variable stiffness joint can be subdivided. A singular perturbation analysis is then applied to the resulting system to show the separation of the dynamics of the different components, that are the arm, the positioning actuators, and the stiffness actuators, obtaining in this way a reduced order model of the manipulator and then a simplified control structure. The proposed control approach is then validated by means of simulations of a three-link planar manipulator with variable stiffness actuation. In this paper, we refer to a variable stiffness joint as a robotic joint actuated by means of a variable stiffness actuator.

\section{System Dynamics}

Starting from the general dynamic model of a robot with $n$ variable stiffness joints as proposed in [10], a second-order dynamic model is used to describe the stiffness behavior of the joints (or, more in general, of the transmissions), also in view of the nature of the mechanisms used to adjust the joint stiffness $[6,10]$.

A scheme of the working principle of a variable stiffness joint is shown in Figure 1: the robot link (on the right side) is moved by means of a positioning actuator (on the left side), and these two parts are connected by an elastic element modulated by means of a second actuator, also referred to as the stiffness actuator (in the lower center part). In [10], it has been shown how the conceptual design shown in Figure 1 can be also used to describe the behavior of antagonistic variable stiffness joints, such as the VSA by Bicchi et al. [12], by means of a suitable change of coordinates in the dynamic model of the device. The overall model of a robotic manipulator equipped with this variable stiffness actuators is then composed by the dynamics of an $n$ DOF arm driven by $n$ positioning actuators through elastic elements and by $n$ stiffness actuators that modify the characteristic of the couplings that connect the positioning actuators to the links. It is assumed that each joint has its own positioning and stiffness actuator and that no direct coupling between the actuators of different joints is present. Let $q, \theta, k \in \mathbb{R}^{n}$ be, respectively, the generalized joint positions, the positions of the the positioning actuators, and the vector of the joint stiffness. Under the simplifying modeling assumption used in [13] (viz., the kinetic energy of the actuators are due only to their own spinning), the dynamic model of the system can be written as

$$
\begin{gathered}
M(q) \ddot{q}+N(q, \dot{q})+K[q-\theta]+\eta_{q}=\tau_{e}, \\
B \ddot{\theta}+K[\theta-q]+\eta_{\theta}=\tau_{\theta}, \\
\gamma(q, \theta, k) \ddot{k}-\beta(q, \theta, k)+\eta_{k}=\tau_{k},
\end{gathered}
$$

where $M(q)$ is the inertia matrix of the robotic arm, the vector $N(q, \dot{q})$ contains the centrifugal, Coriolis, and gravity forces, $K=\operatorname{diag}\left\{k_{1}, \ldots, k_{n}\right\}>0$ is the joint stiffness matrix, $B=\operatorname{diag}\left\{b_{1}, \ldots, b_{n}\right\}>0$ is the inertia matrix of the actuators, $\tau_{\theta}, \tau_{k} \in \mathbb{R}^{n}$ are the positioning and the stiffness actuators command torques, respectively, $\tau_{e}$ is the joint torque contribution due to the effect of an external load; that is,

$$
\tau_{e}=J(q)^{T} F_{e}
$$

where $J(q)$ is the manipulator Jacobian matrix and $F_{e}$ is the external wrench. Equation (3) covers a situation where the stiffness actuation modifies the transmission configuration in order to change its compliance, for example, by precompressing elastic elements or by moving some mechanical parts. As shown in [10], the general model (1)-(3) can be used to describe the characteristics of different variable stiffness joint implementations both in the antagonistic case, such as the VSA proposed by Bicchi et al. [12], and in the nonantagonistic case, such as the device proposed by Wolf and Hirzinger [6], by means of a suitable change of coordinates in the dynamic model of the system. In (3), $\gamma(q, \theta, k)$ and $\beta(q, \theta, k)$ are used to model the stiffness variation as a function of the device configuration, and they depend on the particular implementation of the robot transmission system; refer to [10] for a detailed description of the general model (1)-(3). Note that $K=\operatorname{diag}\left\{k_{1}, \ldots, k_{n}\right\}$ and $k=\left[k_{1}, \ldots, k_{n}\right]^{T}$ are alternative representations of the joint stiffness. It is important to stress that in this paper, the joint stiffness matrix $K$ in (1)-(2) is not constant and that, in general, is a function of time

$$
K=K(t)
$$

as expressed by (3), that models the dynamics of the joint stiffness variation. Moreover, we consider $k_{i}(t)>0$, for all $t$, since it has no physical meaning to consider negative stiffness, while if the stiffness drops to zero, an unactuated system would be obtained.

As a nonrestrictive assumption, all the effects due to frictions, dead zones, nonmodeled dynamics, parameters variations, and so forth, not considered in other terms of the system dynamics (1)-(3), are collected in the functions $\eta_{\{q, \theta, k\}}(t)$. It is then possible to refer to the nominal system dynamics by considering $\eta_{\{q, \theta, k\}}(t)=0$. 


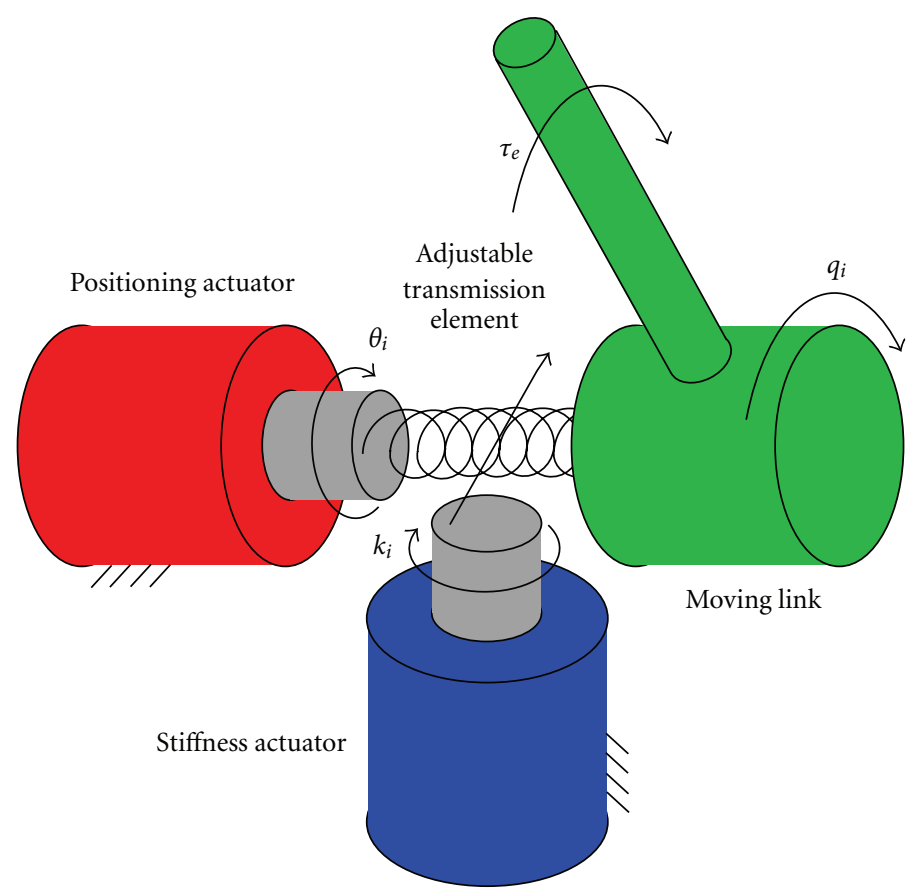

FIGURE 1: Working principle scheme of a variable stiffness joint.

As stated before, the overall system is composed by $3 n$ rigid bodies, and therefore, the state vector of the system can be defined as

$$
\chi=\left[\begin{array}{llllll}
q^{T} & \dot{q}^{T} & \theta^{T} & \dot{\theta}^{T} & k^{T} & \dot{k}^{T}
\end{array}\right]^{T} \in \mathbb{R}^{6 n},
$$

while it is assumed that only the state subset information

$$
\zeta_{m}=\left[\begin{array}{lll}
q^{T} & \theta^{T} & k^{T}
\end{array}\right]^{T} \in \mathbb{R}^{3 n}
$$

are directly measurable, typically by means of optical encoders. Note that the joint stiffness $k$ is usually reconstructed by means of the knowledge of the elastic elements characteristics and by measuring the actuators and joints positions [9].

In any case, the control objective is the simultaneous and independent regulation of the following set of outputs:

$$
\zeta=\left[\begin{array}{l}
q \\
k
\end{array}\right] \in \mathbb{R}^{2 n},
$$

namely, the joint positions (and thus, through the robot direct kinematics, the end-effector pose) and the joint stiffness, acting on the system control inputs

$$
\rho=\left[\begin{array}{c}
\tau_{\theta} \\
\tau_{k}
\end{array}\right] \in \mathbb{R}^{2 n},
$$

by means of an output-feedback controller based on the knowledge of $\zeta_{m}$. As general assumption that takes into account the physical limitations of the system, the state variables are considered bounded; that is, $\|\chi\|<\mathbf{X}$ for some properly defined norm $\|\cdot\|$.

\section{Control Strategy}

In the problem considered here, the main control goal is to achieve the tracking of suitable workspace trajectories assigned to the manipulator end-effector while maintaining a desired level of apparent stiffness. As stated previously, this allows to ensure a harmless behavior of the manipulator in case of collision, in particular with humans.

The term apparent stiffness requires a more detailed discussion. Usually, active stiffness control refers to the case in which the manipulator stiffness is modified by means of the control only and has been introduced in [14]. A more general and notable example of active regulation of both static and dynamic parameters of the manipulator is probably the impedance control [15] that has been successfully used for the control of lightweight robots [16] and for safety evaluation of manipulators [17]. On the other hand, passive stiffness control indicates the possibility of changing the mechanical stiffness of the system. Note that in the case of series-elastic actuators [18, 19], the stiffness is fixed by design and cannot be changed anymore without using an active stiffness control approach. In this paper, the discussion is posed in terms of apparent stiffness, because the desired workspace stiffness is achieved by modulating both the mechanical compliance of the joints, that is, the passive stiffness, and the compliance due to the control. This principle is similar to the combined compliance control [20], and it has been already used also in robotic hands control; see [21].

An important point to note is that in case of an unpredictable impact, only the mechanical stiffness of the manipulator is experienced by the environment (or the human) during the very short time interval in which the energy transfer due to the impact occurs [17]. This is due to the limits 


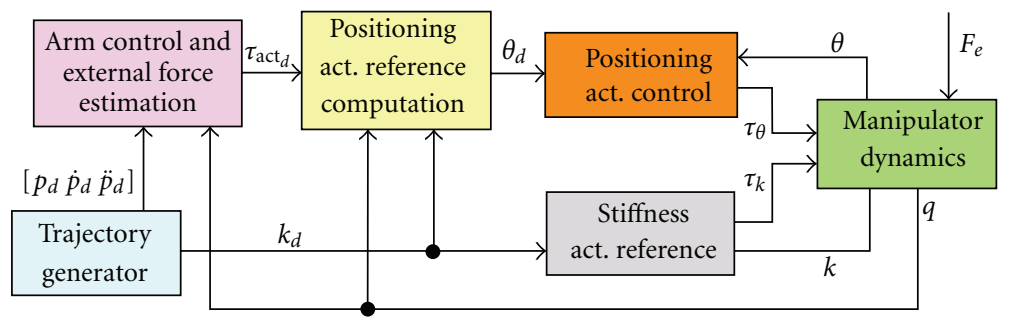

FIGURE 2: Scheme of the overall output-based controller.

in both the sensor and the control bandwidth that prevent an immediate reaction of the control system. Another motivation of this fact is the limited stiffness of the robot structure itself (if compared with the time constant of the force transmission along the structure) and to the fact that the impact may occur in any point along the manipulator, while the torque sensors, when available, are located in the joints. This latter effect, as, in general, the energy exchange from the robot to the environment during impacts, can be attenuated by means of suitable soft covers mounted on the robots [3] that allow early transmission of relatively lower forces along the structure together with some storage and dissipation of the energy due to the impact.

Recalling that variable stiffness actuation has been introduced for safety purposes, and that its main role is to absorb at least part of the kinetic energy of the actuation system (i.e., usually predominant with respect to the one of the arm structure because of the high reduction ratio gearboxes used to connect the actuators to the links) during unpredictable impacts, another important point to note is that the selection of suitable values of the joint stiffness depends mainly on the task to be performed (in particular on the required velocities), on both the manipulator and the actuators inertia, that together with the task specifications determines the overall system kinetic energy, and, the most important, on the criteria used to evaluate the robot safety. To this end, the definition of suitable safety criteria for robotic applications represents a key topic. While in early investigations these criteria have been inherited from automotive industries, it has been successively shown that they are not completely suitable for robotic applications because of the very different scenario [17]. Therefore, great attention has been devoted to the definition of adequate principles for the measurements and the quantification of the robot safety [17]. It is not the topic of this paper to deal with the selection of the joint stiffness: it is here assumed that the joint stiffness trajectory is given by a suitable safety planner. An overview of the complete system is depicted in Figure 2.

3.1. Preliminaries. Consider the generic second-order dynamic system

$$
A(v, w) \ddot{v}+f(\dot{v}, v, w)+\alpha=\tau,
$$

where $v, \dot{v}$ are the state variables, $v$ is the measurable output, $w$ is an exogenous measurable signal, $A(\cdot, \cdot)$ is a bounded symmetric positive definite (invertible) matrix, $f(\cdot, \cdot, \cdot)$ is bounded and Lipschitz in all its arguments, and $\alpha$ is a generic function of the time collecting all the disturbances and uncertainties of the system. It is assumed that

$$
\left\|A^{-1}(v, w)[f(\tilde{v}, v, w)-f(\dot{v}, v, w)]\right\| \leq \sigma\|\tilde{v}-\dot{v}\|, \quad \sigma>0 .
$$

Due to the general separation principle introduced in [22] for SISO systems and then extended to MIMO systems in [23], semiglobal stability (i.e., imposing that the domain of attraction of the equilibrium contains a prescribed compact set, [24]) of the desired equilibrium of (10) in nominal conditions $(\alpha=0)$ can be achieved by output feedback, since (1) state feedback linearization is clearly possible under the previously defined hypotheses and (2) the system is uniform complete observable. While the verification of the former point is trivial, the latter can be easily proven by dynamic extension, [25]. It is straightforward to show that (10) is a generic form that can be used to represent (1)-(3) (see Property 5 in the appendix). Then, the following theorems hold.

Theorem 1. The system (10) in nominal conditions $(\alpha=0)$ and under the effect of the output-based dynamic compensator

$$
\begin{gathered}
\tau=A(v, w)\left\{\bar{\tau}+\Gamma_{3}\left[z_{3}-z_{2}\right]\right\}+f\left(z_{2}, v, w\right), \\
\bar{\tau}=-\Lambda_{1}\left[v-v_{d}\right]-\Lambda_{2}\left[z_{2}-\dot{v}_{d}\right]+\ddot{v}_{d}, \\
\dot{z}_{1}=-\Gamma_{1}\left[z_{1}-v\right]+z_{2}, \\
\dot{z}_{2}=-\Gamma_{2}\left[z_{1}-v\right]+\bar{\tau}, \\
\dot{z}_{3}=\bar{\tau},
\end{gathered}
$$

where $v_{d}$ is the desired value of $v, \Lambda_{1}, \Lambda_{2}, \Gamma_{1}, \Gamma_{2}$, and $\Gamma_{3}$ that are positive definite (diagonal) matrices and has a unique steady-state exponentially stable equilibrium point with arbitrary fast error dynamics.

Proof. By defining the state vector composed by the system tracking error $\varepsilon_{1}$ plus the compensator estimation error $\varepsilon_{2}$ as

$$
\begin{aligned}
\varepsilon & =\left[\left(v-v_{d}\right)^{T}\left(\dot{v}-\dot{v}_{d}\right)^{T} \mid\left(z_{1}-v\right)^{T}\left(z_{2}-\dot{v}\right)^{T}\left(z_{3}-\dot{v}\right)^{T}\right]^{T} \\
& =\left[\varepsilon_{1}^{T} \mid \varepsilon_{2}^{T}\right]^{T},
\end{aligned}
$$

then (10)-(16) can be written as

$$
\dot{\varepsilon}=H \varepsilon+\Theta \hat{\alpha},
$$


where

$$
\begin{aligned}
H & =\left[\begin{array}{cc|ccc}
0 & I & 0 & 0 & 0 \\
-\Lambda_{1} & -\Lambda_{2} & 0 & -\Lambda_{2}-\Gamma_{3} & \Gamma_{3} \\
\hline 0 & 0 & -\Gamma_{1} & I & 0 \\
0 & 0 & -\Gamma_{2} & \Gamma_{3} & -\Gamma_{3} \\
0 & 0 & 0 & \Gamma_{3} & -\Gamma_{3}
\end{array}\right] \\
& =\left[\begin{array}{c|c}
H_{11} & H_{12} \\
\hline 0 & H_{22}
\end{array}\right], \\
\Theta & =\left[\begin{array}{cc|cc}
0 & -I \mid 0 & I & I
\end{array}\right]^{T}=\left[\Theta_{1}^{T} \mid \Theta_{2}^{T}\right]^{T}, \\
\hat{\alpha} & =A^{-1}\left[f(\dot{v}, v, w)-f\left(z_{2}, v, w\right)\right] .
\end{aligned}
$$

The arguments of $A(\cdot, \cdot)$ are omitted for brevity.

At first, the autonomous linear system obtained by neglecting $\hat{\alpha}$ is considered. The eigenvalues of matrix $H$, as can be easily seen by looking at the partitioning in (19), can be freely selected by means of a suitable choice of the parameters $\Lambda_{1}, \Lambda_{2}, \Gamma_{1}, \Gamma_{2}$, and $\Gamma_{3}$.

Due to the linearity of the nominal system under the effect of the proposed controller, the origin is the unique exponentially-stable equilibrium point of the autonomous (nominal) system and the rate of convergence can be made arbitrarily fast.

Now, the implications of the previous hypothesis (11) are considered: on this basis and from (20) it is possible to write

$$
\left\|A^{-1}\left[f\left(z_{2}, v, w\right)-f(\dot{v}, v, w)\right]\right\| \leq \sigma\left\|z_{2}-\dot{v}\right\|, \quad \sigma>0 .
$$

The effects of $f(\cdot)$ on (18) can be seen as a vanishing perturbation of the nominal system, then the exponential stability of the equilibrium point can be recovered by means of a suitable choice of the controller parameters. In particular, by considering a symmetric positive definite matrix $Q$, it is well known that a unique symmetric positive definite matrix $P$ exists that satisfies the Lyapunov equation

$$
P H+H^{T} P=-Q \text {. }
$$

If and only if the matrix $H$ has all negative eigenvalues [26]. The Lyapunov function $V(\varepsilon)=\varepsilon^{T} P \varepsilon$ satisfies the relations

$$
\begin{gathered}
c_{1}\|\varepsilon\|^{2} \leq V(\varepsilon) \leq c_{2}\|\varepsilon\|^{2}, \\
\frac{\partial V}{\partial \varepsilon} H \varepsilon=-\varepsilon^{T} Q \varepsilon \leq-c_{3}\|\varepsilon\|^{2}, \\
\left\|\frac{\partial V}{\partial \varepsilon}\right\|=\left\|2 \varepsilon^{T} P\right\| \leq 2\|P\|\|\varepsilon\|=c_{4}\|\varepsilon\|,
\end{gathered}
$$

where $c_{1}=\lambda_{\min }(P), c_{2}=\lambda_{\max }(P), c_{3}=\lambda_{\min }(Q)$, and $c_{4}=$ $2 \lambda_{\max }(P)$. The derivative of $V(\varepsilon)$ along the trajectory of the system satisfies

$$
\dot{V}(\varepsilon) \leq-c_{3}\|\varepsilon\|^{2}+c_{4} \sigma\|\varepsilon\|^{2},
$$

meaning that the system is exponentially stable if $\sigma<c_{3} / c_{4}$. It is possible to show that this ratio can be maximized by choosing $Q=I[26]$.
Theorem 2. The system (18) under the effects of nonvanishing bounded disturbances $\alpha$

$$
\dot{\varepsilon}=H \varepsilon+\Theta\left[\hat{\alpha}+A^{-1} \alpha\right]
$$

is ultimate uniformly bounded (UUB), [26].

Proof. Theorem 1 shows that the system (18) has the origin $\varepsilon=0$ as unique exponentially stable equilibrium point. It is here supposed that $\|\varepsilon(t)\|<\varsigma$, for all $t \geq 0$. By means of a suitable choice of the control parameters, it possible to ensure that the following condition holds:

$$
\left\|A^{-1} \alpha\right\| \leq \delta<\frac{c_{3}}{c_{4}} \sqrt{\frac{c_{1}}{c_{2}}} \vartheta_{\varsigma},
$$

for some positive constant $\vartheta<1$, where $\delta$ is a positive constant representing a suitable bound of the disturbances. Taking into consideration the same Lyapunov function adopted in Theorem 1, the derivative of $V(\varepsilon)$ along the trajectory of the perturbed system satisfies

$$
\begin{aligned}
\dot{V}(\varepsilon) & \leq-c_{3}\|\varepsilon\|^{2}+c_{4} \sigma\|\varepsilon\|^{2}+c_{4} \delta\|\varepsilon\| \\
& =-[1-\vartheta]\left[c_{3}-\sigma c_{4}\right]\|\varepsilon\|^{2}-\vartheta\left[c_{3}-\sigma c_{4}\right]\|\varepsilon\|^{2}+c_{4} \delta\|\varepsilon\| \\
& \leq-[1-\vartheta]\left[c_{3}-\sigma c_{4}\right]\|\varepsilon\|^{2}, \quad \forall\|\varepsilon\| \geq \frac{c_{4} \delta}{\vartheta\left[c_{3}-\sigma c_{4}\right]}
\end{aligned}
$$

Then, for all $\left\|\varepsilon\left(t_{0}\right)\right\|<\sqrt{c_{1} / c_{2}} \varsigma$, the trajectory of the system satisfies the relations

$$
\begin{array}{r}
\|\varepsilon(t)\| \leq \sqrt{\frac{c_{2}}{c_{1}}} \exp \left\{-\frac{[1-\vartheta]\left[c_{3}-\sigma c_{4}\right]}{c_{4}}\left[t-t_{0}\right]\right\}\left\|\varepsilon\left(t_{0}\right)\right\|, \\
\forall t_{0} \leq t<t_{0}+T, \\
\|\varepsilon(t)\| \leq \frac{c_{4}}{c_{3}-\sigma c_{4}} \sqrt{\frac{c_{2}}{c_{1}}} \frac{\delta}{\vartheta}, \forall t \geq t_{0}+T,
\end{array}
$$

for some finite time interval $T$, see [26] for additional details on the proof.

Property 1. Due to the structure of $H$ and to the partitioning of $\varepsilon$, the eigenvalues of the dynamic compensator (i.e., of matrix $H_{22}$ ) depend only on $\Gamma_{1}, \Gamma_{2}$ and $\Gamma_{3}$, while those of the controlled system (i.e., of $H_{11}$ ) depend on $\Lambda_{1}$ and $\Lambda_{2}$. This separation property is a consequence of the system linearization obtained by output feedback.

Property 2. Note that (16) represents the internal model of the system, and by assuming constant values of $\alpha$, namely, $\alpha_{\text {ss }}$, the steady-state equilibrium point of the system is

$$
\varepsilon_{\mathrm{ss}}=-H^{-1} \Theta \alpha_{\mathrm{ss}}=\left[\begin{array}{lllll}
0 & 0 & 0 & 0 & \Gamma_{3}^{-1^{T}}
\end{array}\right]^{T} \alpha_{\mathrm{ss}},
$$

thus resulting that only $z_{3}$ is affected by the external disturbance. 
Generally speaking, by applying a singular perturbation analysis, from Property 1 , and by assuming that the eigenvalues of $H_{22}$ are fast enough with respect to those of $H_{11}$, it is possible to assume that the system

$$
\dot{\varepsilon}_{2}=H_{22} \varepsilon_{2}+\Theta_{2}\left[\hat{\alpha}+A^{-1} \alpha\right]
$$

is always working in steady-state conditions, and its equilibrium point is

$$
\varepsilon_{2}=-H_{22}^{-1} \Theta_{2} A^{-1} \alpha_{s s} .
$$

This last relation is a generalization of (29). It follows that

$$
z_{1} \longrightarrow v, \quad z_{2} \longrightarrow \dot{v}, \quad z_{3}-z_{2} \longrightarrow \Gamma_{3}^{-1} A^{-1} \alpha_{\mathrm{ss}}
$$

Then, $z_{3}$ (together with $z_{2}$ ) provides a filtered estimation of the disturbance. This effect on the system can be seen as a disturbance compensation action (i.e., it compensates for the effects of $\alpha$ on the system state), and this compensation action can be annihilated by posing $\Gamma_{3}=0$ in (13)-(16). Note that from (19), this is a singular condition for the controller.

Property 3. The control system (10)-(16) can be used also as a regulator by neglecting the time derivative of the reference signal $\dot{v}_{d}$ and $\ddot{v}_{d}$. This property will be exploited for the design of the fast actuators controllers, since, in this case, tracking performance are not necessary, and these controllers are assumed fast enough to work always in steady-state conditions with respect to the slow arm controller.

Property 4. By redefining the control action in (13) as

$$
\bar{\tau}=-\Lambda_{1}\left[v-v_{d}\right]-\Lambda_{2}\left[z_{2}-\dot{v}_{d}\right]+\ddot{v}_{d}+u,
$$

where $u$ is an auxiliary input signal, the system dynamics becomes

$$
\begin{gathered}
\dot{\varepsilon}=H \varepsilon+G u+\Theta\left[\hat{\alpha}+A^{-1} \alpha\right], \\
G=\left[\begin{array}{lllll}
0 & 1 & 0 & 0 & 0
\end{array}\right]^{T} .
\end{gathered}
$$

Due to the structure of matrix $G$, it is possible to note that the auxiliary input $u$ does not influence the compensator error dynamics $\varepsilon_{2}$, and therefore, the resulting dynamics of the tracking error $\varepsilon_{1}$ is

$$
\dot{\varepsilon}_{1}=\left[\begin{array}{cc}
0 & I \\
-\Lambda_{1} & -\Lambda_{2}
\end{array}\right] \varepsilon_{1}+\left[\begin{array}{l}
0 \\
I
\end{array}\right] u,
$$

showing a steady state tracking error $\left(v-v_{d}\right)=\Lambda_{1}^{-1} u$.

3.2. Actuators Control Design. It is important to note that both (2) and (3) are in the form of (10) (see Property 5 in the appendix). This essentially means that both the positioning and the stiffness actuators dynamics can be decoupled from the arm dynamics (1) by exploiting the properties of the controller (12)-(16). Even if this decoupling can be achieved by means of a unique controller, the use of two separated controllers, one for the positioning actuators and one for the stiffness actuators, is chosen to have a more clear view of the overall system structure. Due to the implications of Theorem 1, the possibility of assigning an arbitrary dynamics to the actuators allows to obtain a two time-scale system, where the actuators represent the fast system and the arm the slow one, and then to apply a singular perturbation approach to reduce the order of the resulting system

$$
\begin{gathered}
M(q) \ddot{q}+N(q, \dot{q})+K[q-\theta]+\eta_{q}=\tau_{e}, \\
\dot{\varepsilon}_{\theta}=H_{\theta} \varepsilon_{\theta}+\Theta_{\theta}\left[\hat{\alpha}_{\theta}+B^{-1} \alpha_{\theta}\right], \\
\dot{\varepsilon}_{k}=H_{k} \varepsilon_{k}+\Theta_{k}\left[\hat{\alpha}_{k}+\gamma^{-1} \alpha_{k}\right],
\end{gathered}
$$

where

$$
\begin{gathered}
\varepsilon_{\theta}=\left[\left(\theta-\theta_{d}\right)^{T} \dot{\theta}^{T} \mid\left(z_{1_{\theta}}-\theta\right)^{T}\left(z_{2_{\theta}}-\dot{\theta}\right)^{T}\left(z_{3_{\theta}}-\dot{\theta}\right)^{T}\right]^{T}, \\
\varepsilon_{k}=\left[\left(k-k_{d}\right)^{T} \dot{k}^{T} \mid\left(z_{1_{k}}-k\right)^{T}\left(z_{2_{k}}-\dot{k}\right)^{T}\left(z_{3_{k}}-\dot{k}\right)^{T}\right]^{T},
\end{gathered}
$$

and $\theta_{d}$ is the vector of the desired positioning actuators configuration, $k_{d}=\left[k_{1_{d}}, \ldots, k_{n_{d}}\right]$ is the vector of the desired joint stiffness, and $\hat{\alpha}_{\{\theta, k\}}, \alpha_{\{\theta, k\}}$ summarize the disturbances acting on the positioning and stiffness actuators, respectively. In the case of the actuators controllers, Property 3 has been exploited, since, with respect to the arm dynamics, these controllers are always working in steady-state conditions, then tracking performance are not necessary but just the regulation of the desired position and stiffness. Note that the robotic arm dynamics remains the same as in (1). On the basis of Theorem 1, for both (36) and (37), it is possible to write

$$
H^{-1} \dot{\varepsilon}=\varepsilon+H^{-1} \Theta \hat{\alpha},
$$

where the distinction between the positioning and the stiffness actuators has been omitted for brevity. As long as the eigenvalues of $H$ can be freely assigned, it is clear how the time scale of (36) and (37) can be made arbitrarily faster than the time scale of (1); therefore, (39) represents the fast manifold dynamics. With some abuse of notation, as $\left\|H^{-1}\right\| \rightarrow 0$ (remember that this can be achieved by a suitable choice of the controller parameters) the fast manifold dynamics (39) degenerates, and due to Theorem 1, it is possible to state that the fast manifold has a unique root expressed by (29). From (29)-(37), since the disturbance does not affect the coupling between the actuators and the arm, it is then possible to define the reduced-order model of the robotic manipulator with variable joint stiffness as

$$
M(q) \ddot{q}+N(q, \dot{q})+K_{d}\left[q-\theta_{d}\right]+\eta_{q}=\tau_{e},
$$

where the actual actuator configuration $\theta$ and $K$ have been substituted with the desired ones $\theta_{d}$ and $K_{d}$ in the arm dynamic equation.

3.3. Trajectory Planning for the Actuators. In this section, the problem of defining the desired trajectories for the actuators 
given the manipulator trajectories, both in terms of position and stiffness, is addressed. Note that in case the desired manipulator stiffness is defined in the joint space only, the stiffness actuator reference signals are fully defined by $K_{d}$, then the more general case of workspace stiffness reference is here addressed.

It is important to note that once the desired joint stiffness $K_{d}$ has been determined, the torque provided to the joints by the actuators can be regulated by acting on $\theta_{d}$, that is, by a suitable selection of the positioning actuators configuration. To this end, (40) can be rewritten as

$$
\begin{gathered}
M(q) \ddot{q}+N(q, \dot{q})+\eta_{q}=\tau_{e}+\tau_{\mathrm{act}}, \\
\tau_{\mathrm{act}}=K_{d}\left[\theta_{d}-q\right]
\end{gathered}
$$

where $\tau_{\text {act }}$ is the vector of the torques provided by the arm virtual actuators obtained from the joined action of the stiffness and the positioning actuators. It follows that

$$
\theta_{d}=K_{d}^{-1} \tau_{\mathrm{act}_{d}}+q
$$

where $\tau_{\mathrm{act}_{d}}$ is the desired torque to be applied on the arm joints by the actuation system and computed by the arm controller that will be defined in the following.

3.3.1. Position Trajectory. Let us define the direct kinematic problem and the manipulator Jacobian as

$$
p=\Psi(q) \longrightarrow \dot{p}=\frac{\partial \Psi(q)}{\partial q} \dot{q}=J(q) \dot{q}
$$

where $p \in \mathbb{R}^{m}$ is the manipulator end-effector position in an $m$-dimensional task space. Differently from the case of state feedback linearization [10], where $q_{d} \in \mathbb{C}^{4}$ and $k_{d} \in \mathbb{C}^{2}$, that is, the joint trajectories must be defined up to the fourth time derivative, while the joint stiffness must be defined up to the second time derivative, in this case, due the reduced order of the arm dynamics (40), the desired manipulator trajectory can be specified as usual for rigid robots both in the task space and in the joint space. In other words, the robot trajectory will be defined in terms of desired positions, velocities, and accelerations, while no particular requirements are necessary for the stiffness desired values. In any case, smooth-enough stiffness profiles are preferable to avoid abrupt oscillations of the actuators and of the arm.

If the manipulator trajectory is defined in the task space, the vectors $p_{d}, \dot{p}_{d}$, and $\ddot{p}_{d}$ indicate, respectively, the desired position, velocity, and acceleration of the end effector. It is assumed that both task- and joint-space trajectories are limited and continuous (at least with a piece-wise continuous acceleration profile). Obviously, in case of task space control, singular configurations of the manipulator must be avoided or explicitly considered in the controller. In that case, it is then possible to define the desired joint-space trajectory as

$$
\begin{gathered}
q_{d}=\Psi^{-1}\left(p_{d}\right), \\
\dot{q}_{d}=J\left(q_{d}\right)^{\#} \dot{p}_{d}, \\
\ddot{q}_{d}=J\left(q_{d}\right)^{\#}\left[\ddot{p}_{d}-\dot{J}\left(q_{d}\right) \dot{q}_{d}\right],
\end{gathered}
$$

where (45) indicates the inverse kinematic function. The solution of the manipulator inverse kinematics can be also obtained by means of a closed-loop inverse kinematic algorithm (CLIK), [27], as specified in the following. In case of manipulator redundancy $(n>m)$, the Jacobian pseudoinverse $J\left(q_{d}\right)^{\#}$ must be considered. Note that the manipulator redundancy can be exploited by defining secondary tasks [28], for example, to avoid obstacles, to deal with general task constraints, or to optimize the manipulator joint configuration on the basis of the desired task space stiffness, as will be shown in the following.

3.3.2. Stiffness Reference Signals. In the following discussion, the leading superscript ${ }^{w}$ will be used to refer to workspacerelated variables. In static conditions, the workspace stiffness of the arm ${ }^{w} K_{d}$ can be specified as

$$
F_{e}={ }^{w} K_{d} d p,
$$

where the infinitesimal end-effector workspace displacement $d p$ and the environmental force $F_{e}$ are considered.

Several works can be found in the literature about the workspace stiffness control of both redundant and nonredundant robots. In particular, in [29], the different components that determine the total workspace stiffness are analyzed, and the orthogonal stiffness decomposition control is introduced, while in [30], the stability properties of the workspace stiffness components and a minimal parametrization of the manipulator stiffness and compliance are reported. Since variable stiffness actuation allows the independent control of the joint compliance, an interesting approach for the regulation of the workspace compliance of redundant robots with compliant actuation can be the combined control approach proposed in [20], where the desired manipulator stiffness in the operational space is achieved by combining a decoupled stiffness joint control together with a workspace stiffness controller.

The relation between the workspace stiffness ${ }^{w} K_{d}$ and the overall joint stiffness $K_{a}$ can be expressed as

$$
K_{a}=J^{T}{ }^{w} K_{d} J
$$

where the dependence of the Jacobian from the joint configuration $q$ has been omitted for brevity. Note that the overall joint stiffness $K_{a}$ may be the result of several effects, as specified in the following. For this reason, this quantity has been distinguished from the mechanical joint stiffness $K_{d}$. In the right-hand side of (48), since the workspace stiffness must be, in general, symmetric and positive definite and due to the transformation through the manipulator Jacobian, only $m[m+1] / 2$ independent terms are present. At the same time, by considering the case of independent regulation of the joint mechanical stiffness only, matrix $K_{a}$ is diagonal $\left(K_{a}=K_{d}\right)$, then there are $n$ independent terms in the left-hand side of (48). It follows that it is possible to assign arbitrary workspace stiffness by selecting suitable joint mechanical stiffness only if $n=m[m+1] / 2$, that means a certain redundancy in the manipulator, at least nonconsidering the trivial case $m=1$, that is, a single DOF mechanism. If $n>$ $m[m+1] / 2$, there exist infinite combinations of joint stiffness 
that allow to achieve the desired workspace stiffness. Then, this redundancy must be managed by suitable projection of the workspace stiffness on the joint space [29], and it can be exploited maybe to minimize (or maximizing depending on the task) the overall joint stiffness. If $n<m[m+1] / 2$, it is not possible to control all the components of the workspace stiffness be means of the joint stiffness only, and suitable couplings between the components of the workspace stiffness must be introduced.

It is now important to remark that the introduction of variable stiffness joints is useful to reduce the energy transferred from the manipulator to the environment (or humans) during unpredictable impacts by decoupling the actuators inertia from the link during fast robot motions (i.e., by reducing the joint stiffness) or, on the other hand, to increase the precision of the manipulator during slow and limited movements by imposing high joint stiffness values to allow adequate external disturbance force/torque rejection. Moreover, variable stiffness actuators are usually designed to have high compliance in rest conditions and to become rigid if necessary under the action of the control system. In this sense, it is then clear that the role of the joint mechanical stiffness, or passive stiffness, is completely different from the active stiffness, that is a control strategy that to allow to rigid robots to show a "compliant" behavior with respect to environmental uncertainties, for example, during assembly operations [14].

By introducing the concept of combined compliance control reported in [20] but with different goals, it is possible to split the overall workspace stiffness into two components, one given by the mechanical joint stiffness $K_{d}$ only, the passive stiffness, that acts at all the frequencies but that shows its major effects at high frequency and then in case of impacts, and in a component due to the control, the active stiffness $K_{c}$, that is significant only within the control bandwidth. It is also important to remark that due to the cascade structure of the proposed controller, it is possible to design the arm controller with a purposely defined bandwidth to decouple the passive stiffness from the active stiffness.

The contribution to the workspace stiffness due to the mechanical joint stiffness $K_{d}$ only, namely, ${ }^{w} K_{j}$, is given by

$$
{ }^{w} K_{j}^{-1}=J K_{d}^{-1} J^{T} .
$$

It is important to note that (49) maps the joint stiffness to the workspace stiffness no matter what is the number of joints or the dimension of the workspace. This point is important in case of manipulator redundancy to deal with suitable selection of the joint stiffness.

On the basis of these considerations, the workspace stiffness ${ }^{w} K_{d}$ can be partitioned into two different contributions

$$
{ }^{w} K_{d}={ }^{w} K_{j}+{ }^{w} K_{c},
$$

where ${ }^{w} K_{j}$ is the contribution due to the passive mechanical joint stiffness and ${ }^{w} K_{c}$ is the active stiffness due to the control. Note that since from (49) ${ }^{w} K_{j}$ is symmetric positive definite by definition and ${ }^{w} K_{c}$ must be symmetric and positive definite to avoid instability of the positioning actuators, also ${ }^{w} K_{d}$ is positive definite, thus avoiding system instability: this results in a lower limit of the workspace stiffness given by the effects of the joint mechanical stiffness, since the controller can be used only to increment the overall stiffness. It is important to remark that while in the case of industrial robots the control objective is to make rigid robot softer and then the system compliance is given by the sum of the control compliance and the joint compliance, this last equation shows that the proposed control system is used to the make soft robots (because of the relatively low value of

${ }^{w} K_{j}$ ) stiffer when necessary by means of control (i.e., by acting on ${ }^{w} K_{c}$ ).

From (49) and (50), the induced partitioning of the apparent joint stiffness $K_{a}$ is

$$
K_{a}=K_{d}+J^{T}{ }^{w} K_{c} J=K_{d}+K_{c}
$$

Due to the effect of the joint apparent stiffness, the relation between the infinitesimal joint displacements and the joint torques can be expressed as

$$
\tau_{\mathrm{act}_{d}}=K_{a} d q
$$

Taking into consideration the relation between the workspace displacement and joint displacement, by inverting (52), it is possible to write

$$
\begin{aligned}
d q & =K_{a}^{-1} \tau_{\mathrm{act}_{d}}=K_{a}^{-1} J^{T} F_{e} \\
& =K_{a}^{-1} J^{T}{ }^{w} K_{d} d p=K_{a}^{-1} J^{T}\left[J K_{a}^{-1} J^{T}\right]^{-1} d p
\end{aligned}
$$

where the relation between the workspace stiffness and the joint apparent stiffness ${ }^{w} K_{d}{ }^{-1}=J K_{a}^{-1} J^{T}$ has been exploited (see Theorem 3 in the appendix). It is possible to demonstrate that the same relation can be written in the form (Theorem 3 in the appendix) [20]

$$
d q=K_{d}^{-1} J^{T}\left[J K_{d}^{-1} J^{T}\right]^{-1} d p=J^{\#} d p
$$

where $J^{\#}=K_{d}^{-1} J^{T}\left[J K_{d}^{-1} J^{T}\right]^{-1}$ is the pseudoinverse Jacobian matrix, weighted by the joint mechanical stiffness $K_{d}$. This result is important, because it shows that the relations between workspace and joint displacements are affected only by the mechanical joint stiffness. From the meaning of the pseudoinverse, the inverse kinematic solution obtained from (54) can be considered as providing the manipulator configuration which minimizes the deviation of the joints weighted by the joint stiffness; that is, it minimizes the potential energy stored in the joint compliance, ensuring the minimum energy transfer due to the inertia of the actuators during unexpected collisions. It follows that the desired workspace stiffness has no effect on the inverse kinematics solution of the manipulator. This fact suggests that the regulation of the manipulator configuration $q$ must be performed on the basis of the inverse kinematic solution obtained by means of $J^{\#}$ both to stabilize the manipulator dynamics in the Jacobian null space and to achieve minimum energy transfer from the actuators to the arm. 
3.4. Arm Control Design. Several robust controllers for robotic arms have been presented in the literature; see for example, [31-33] for some well-known examples. Anyway, in case of robots with variable joint stiffness, the application suggests a particular implementation of the controller.

First of all, aiming to clarify the role of the passive and the active stiffness, the static relation between the environmental force $F_{e}$ and the deviation of the end effector in the workspace is considered

$$
F_{e}={ }^{w} K_{d}\left[p_{d}-p\right] \Longrightarrow \tau_{\mathrm{act}_{d}}=J^{T} F_{e}=J^{T}{ }^{w} K_{d}\left[p_{d}-p\right]
$$

and for small-enough displacements,

$$
\tau_{\mathrm{act}_{d}}=J^{T}{ }^{w} K_{d} J\left[q_{d}-q\right]=K_{a}\left[q_{d}-q\right] .
$$

From (43) and (51), it follows that

$$
\begin{aligned}
\theta_{d} & =K_{d}^{-1} K_{a}\left[q_{d}-q\right]+q=K_{d}^{-1}\left[K_{d}+K_{c}\right]\left[q_{d}-q\right]+q \\
& =q_{d}+K_{d}^{-1} K_{c}\left[q_{d}-q\right] .
\end{aligned}
$$

By substituting (57) into (41)-(42), it follows that

$$
M(q) \ddot{q}+N(q, \dot{q})+\underbrace{\left[K_{d}+K_{c}\right]}_{K_{a}}\left[q-q_{d}\right]+\eta_{q}=\tau_{e},
$$

that means that the robotic arm behaves as a joints-position controlled system with the desired apparent stiffness.

Looking at the right hand side of (57), the first term represents the passive stiffness, since the positioning actuators are commanded just on the basis of the manipulator joint desired position trajectory, and then, no feedback from the actual joint positions is present, while the second term represents the active stiffness, since it implies the joint positions feedback on the positioning actuators. It is important to remark that while the passive stiffness acts at high frequency since it does not require the intervention of any controller, the active stiffness acts within the arm controller bandwidth. Moreover, if the manipulator configuration is obtained by the inverse kinematic solution by using (54), the minimum value for the potential energy stored in the joints as a consequence of the end-effector displacement is achieved.

With the aim of controlling the workspace motion of the manipulator, a specialized version of the controller (12)(16) that in particular exploits Property 4 is now applied to the arm reduced model (41)-(43) (see Property 6 in the appendix). The torque of the virtual actuators can be then computed as

$$
\begin{aligned}
& \tau_{\text {act }_{d}}=M(q)\left\{J^{\#[}\left[\bar{F}-j \bar{z}_{2}\right]+\tau_{j}\right\} \\
& +N\left(q, \bar{z}_{2}\right)-J^{T} F_{e_{m}}+M(q) \bar{\Gamma}_{3}\left[\bar{z}_{3}-\bar{z}_{2}\right], \\
& \bar{F}={ }^{w} M_{d}{ }^{-1}\left\{-{ }^{w} K_{d}\left[p-p_{d}\right]\right. \\
& \left.-{ }^{w} D_{d}\left[J \bar{z}_{2}-\dot{p}_{d}\right]+{ }^{w} M_{d} \ddot{p}_{d}+\hat{F}_{e}\right\}, \\
& \tau_{j}=S\left\{-K_{d}\left[q-q_{d}\right]-D_{d}\left[\bar{z}_{2}-\dot{q}_{d}\right]\right\}, \\
& \dot{\bar{z}}_{1}=-\bar{\Gamma}_{1}\left[\bar{z}_{1}-q\right]+\bar{z}_{2} \text {, }
\end{aligned}
$$

$$
\begin{gathered}
\dot{\bar{z}}_{2}=-\bar{\Gamma}_{2}\left[\bar{z}_{1}-q\right]+J^{\#[}\left[\bar{F}-j \bar{z}_{2}\right]+\tau_{j}, \\
\dot{\bar{z}}_{3}=J^{\#}\left[\bar{F}-j \bar{z}_{2}\right]+\tau_{j},
\end{gathered}
$$

where $S=I-J^{\#} J$ is the matrix that projects the joint space control action in the null space of the manipulator Jacobian, $D_{d}=2 \sqrt{K_{d}}$ is the joint space damping, ${ }^{w} D_{d}$ and ${ }^{w} M_{d}$ represent the desired workspace damping and mass of the manipulator, respectively, and $\bar{\Gamma}_{1}, \bar{\Gamma}_{2}, \bar{\Gamma}_{3}$ are positive definite (diagonal) matrices. In particular, $q_{d}$ and $\dot{q}_{d}$ are provided by a closed-loop inverse kinematic solver that exploits (54), while $F_{e_{m}}$ and $\hat{F}_{e}$ are the external force measurement and estimation, respectively. On the basis of Property 4, the external force estimation is used as the auxiliary input $u$ appearing in (34) in the arm controller. Note that on the basis of (54), the static term $K_{d}\left[q-q_{d}\right]$ in the joint space control ensures the minimum norm of the joint space control effort.

It is initially assumed that the force $F_{e}$ applied by the end effector to the environment is measurable (i.e., by means of a 6-axis force/torque sensor), then it is possible to impose $F_{e_{m}}=\hat{F}_{e}=F_{e}$. Note that by recalling the boundedness of the arm trajectory and the limited value of the state variables and in particular of both the joint velocity $\dot{q}$ and of the controller state $\bar{z}_{2}$, the condition (11) is ensured by the general properties of the robotic arm dynamics $[34,35]$.

By recalling Property 2, and with a suitable choice of large-enough values of $\bar{\Gamma}_{\{1,2,3\}}$, the dynamic compensator represented by (62)-(64) can be configured as a third fast system together with the actuators controllers previously defined. Due to the controller structure, the arm response would be slower in two-order than the actuation control system. This fact does not represent a limitation, since (1) the fast control systems are very simple, and then, very fast implementations are possible with current technologies and computational capabilities of modern digital systems, (2) robots with variable stiffness actuation are conceived for the interaction with humans and to operate in a humanlike environment, then smoother and bandwidth-limited motions are required with respect to industrial robots, (3) fast high-gain controllers are used in the actuators, while a slow low-gain controller is more suitable for the arm control also for safety reasons. It is then possible to assume that

$$
\bar{z}_{1} \longrightarrow q, \quad \bar{z}_{2} \longrightarrow \dot{q}, \quad \bar{z}_{3}-\bar{z}_{2} \longrightarrow \bar{\Gamma}_{3}^{-1} \bar{\alpha},
$$

where $\bar{\alpha}=M(q)^{-1} \eta_{q}$ is the effect of the model and parameter uncertainties. Then, by denoting $e=p-p_{d}$, the reducedorder nominal arm dynamics (41) under the effect of the controller (59)-(60) can be written as

$$
{ }^{w} M_{d} \ddot{e}+{ }^{w} D_{d} \dot{e}+{ }^{w} K_{d} e=F_{e} .
$$

In case a direct measure of the force applied by the end effector to the environment is not available, $\left(F_{e_{m}}=0\right)$ by assuming the perfect knowledge of the arm dynamics, that is, $\eta_{q}=0$, and by considering the external force $F_{e}$ as a disturbance, Property 2 implies that in steady state conditions,

$$
\bar{z}_{1} \longrightarrow q, \quad \bar{z}_{2} \longrightarrow \dot{q}, \quad \bar{z}_{3}-\bar{z}_{2} \longrightarrow \bar{\Gamma}_{3}^{-1} M(q)^{-1} J^{T} F_{e} .
$$


A suitable estimation of the external force $F_{e}$ can be then assumed as

$$
\widehat{F}_{e}=-J^{\#^{T}} M(q) \bar{\Gamma}_{3}\left[\bar{z}_{3}-\bar{z}_{2}\right] .
$$

This equation defines a virtual force sensor that provides an indirect measure of the external force. Hence, the reducedorder nominal arm dynamics can be written as

$$
{ }^{w} M_{d} \ddot{e}+{ }^{w} D_{d} \dot{e}+{ }^{w} K_{d} e=\widehat{F}_{e}+{ }^{w} M_{d} J M(q)^{-1} J^{T}\left[F_{e}-\widehat{F}_{e}\right] .
$$

The last term in (69) represents the perturbation on the system dynamics due to the use of the force estimation instead of a direct measure. Apart from this spurious term, the robotic manipulator is now configured as an impedance controlled system.

Once the torque $\tau_{\text {act }_{d}}$ is determined by means of (59), the reference trajectory for the positioning actuators can be computed through (43).

An important remark is that while the workspace stiffness ${ }^{w} K_{d}$ is obtained by combining the mechanical stiffness of the robot transmission together with the control action, only the active part of the desired stiffness implies the feedback of the manipulator joint positions. This is a key feature for safety reasons [2], because no reaction from the controller is needed to achieve the desired compliant behavior at joint level, since the compliance is a structural characteristic of the robot itself.

Note that also the arm controller provides a filtered estimation of the disturbance acting on the joints through (64). These information, also called residuals in the robotic community [36], can be used for fault evaluation and collision detection/reaction [37], and they have recently been successfully used for safe interaction of lightweight robots with human operators [17]. The possibility of exploiting the properties of robots with variable stiffness actuation together with the safety-oriented control techniques related to the use of the residuals justifies the proposed implementation of the controller.

3.5. Remarks. With respect to the control approaches for the system (1)-(3) proposed in [10,11], the control scheme presented in this paper presents a number of remarkable advantages

(i) The complexity of the controller is significantly reduced since neither static nor dynamic inversion of the system is needed.

(ii) Redundancy of the manipulator is considered.

(iii) The computation of the Lie derivative of the system dynamics up to the fourth order is avoided.

(iv) An output-feedback control law has been defined instead of state-feedback controllers.

(v) The arm trajectory must now be defined only up to the second order derivative, as in the rigid robot case, while previously, it was necessary to define the position trajectory up to the fourth order and the stiffness trajectory to the second order derivative. (vi) Since the arm control is based on the two signals $\theta_{d}$ and $K_{d}$, that are generated online and that act as exogenous reference for the actuators fast system, online compensation of disturbances is permitted.

(vii) The joint stiffness is controlled by a specific control system that directly compensates for the effects of disturbances, external forces and couplings due to both positioning actuators and arm dynamics.

(viii) The proposed control strategy exploits the mechanical compliance of the transmission to achieve the desired workspace behavior (passive compliance).

(ix) In case of perfect knowledge of the arm dynamics, the system is able to estimate and regulate the workspace interaction force without a direct measurement of the force itself.

(x) The manipulator residuals are directly provided by the controller, allowing a simple integration with fault and collision detection/reaction techniques.

(xi) The extension of the proposed approach to the case of robotic manipulator where the stiffness can be adjusted only for some joints is straightforward, since by applying the proposed positioning and stiffness actuators controller where necessary, the overall robot dynamics is reduced to the rigid case.

\section{Simulation of a Three-Link Planar Manipulator}

The proposed controller has been validated by means of simulations of a planar three-link robotic arm with variable joint stiffness. Only the Cartesian coordinates $x$ and $y$ of the workspace end-effector position are considered (not the orientation), and this allows to analyze the effects of the manipulator redundancy in a quite simple manner. The extension of the proposed control approach to the case in which gravity compensation is needed is straightforward. The well-known dynamic model of the three-link planar arm is omitted for brevity; see [35], and only the dynamics of stiffness variation is specified

$$
\lambda_{2} \ddot{k}_{i}+\lambda_{1} k_{i}^{2}+\lambda_{0}\left[q_{i}-\theta_{i}\right]^{2}+\eta_{k_{i}}=\tau_{k_{i}} .
$$

This stiffness dynamic model [10] is typical of a variable stiffness actuator with antagonistic elastic elements characterized by quadratic force-deformation relation proposed by Migliore et al. in [5]. The parameters of the three-link planar manipulator used in simulation are reported in Table 1, and all the links of the manipulator are considered identical for simplicity. In the simulation scheme, the trajectories are generated using proper filters to compute the position trajectory in the workspace together with its derivatives up to the second order. In this manner, the conditions given by Theorem 1 for the stability of the system are implicitly met. It is assumed that the joint stiffness reference values are provided by a suitable safety controller on the basis of some requirements given by the particular application 
TABLE 1: Nominal parameters of the three-link planar manipulator.

\begin{tabular}{lcc}
\hline Description & Value & Unit \\
\hline Joint inertia & $1.15 e-2$ & $\mathrm{Kg} \mathrm{m}^{2}$ \\
Joint viscous friction coeff. & 0.001 & $\mathrm{~N} \mathrm{~s} \mathrm{~m}^{-1}$ \\
Link mass & 0.541 & $\mathrm{~kg}$ \\
Link center of mass & 0.085 & $\mathrm{~m}$ \\
Link length & 0.3 & $\mathrm{~m}$ \\
Motors inertia & $6.6 e-5$ & $\mathrm{~kg} \mathrm{~m}^{2}$ \\
Motors viscous friction coeff. & 0.00462 & $\mathrm{~N} \mathrm{~s} \mathrm{~m}^{-1}$ \\
Actuators Coulomb friction level & 0.1 & $\mathrm{~N} \mathrm{~m}$ \\
Joints Coulomb friction level & 0.5 & $\mathrm{~N} \mathrm{~m}^{2}$ \\
$\lambda_{2}$ & 2.2 & $\mathrm{~s}^{2}$ \\
$\lambda_{1}$ & 0.4 & $\mathrm{~N}^{-1} \mathrm{~m}^{-1}$ \\
$\lambda_{0}$ & 14.7 & $\mathrm{~N} \mathrm{~m} \mathrm{rad}^{-2}$ \\
\hline
\end{tabular}

TABLE 2: Parameters of the three-link planar manipulator controller used in the simulations.

\begin{tabular}{lcc}
\hline Symbol & Case no. 1 & Case no. 2, no. 3 \\
\hline${ }^{w} K_{d}$ & $\operatorname{diag}\{16,16\}$ & $\operatorname{diag}\{100,100\}$ \\
${ }^{w} D_{d}$ & $\operatorname{diag}\{6,6\}$ & $\operatorname{diag}\{30,30\}$ \\
${ }^{w} M_{d}$ & $\operatorname{diag}\{0.02,0.02\}$ & $\operatorname{diag}\{0.02,0.02\}$ \\
$\bar{\Gamma}_{1_{i}}$ & 90 & 90 \\
$\bar{\Gamma}_{2_{i}}$ & $2.7 e 3$ & $2.7 e 3$ \\
$\bar{\Gamma}_{3_{i}}$ & 10 & 10 \\
\hline
\end{tabular}

TABLE 3: parameters of the positioning and stiffness actuators fast controllers used in the simulations.

\begin{tabular}{lc}
\hline Symbol & Case no. 1, no. 2, no. 3 \\
\hline$\Lambda_{1_{i}}$ & $1 e 4$ \\
$\Lambda_{2_{i}}$ & 150 \\
$\Gamma_{1_{i}}$ & $9 e 3$ \\
$\Gamma_{2_{i}}$ & $2.7 e 5$ \\
$\Gamma_{3_{i}}$ & $1 e 3$ \\
\hline
\end{tabular}

and contest. Moreover, the matrices that characterize the arm impedance controller have been considered diagonal to simplify the analysis of the simulation results. The controller parameters adopted in the simulations are summarized in Tables 2 and 3. Three case studies have been considered: in the first case, whose results are reported in Figure 3, the manipulator end-effector is driven along several pointto-point trajectories. During this simulation, friction in the actuators is considered together with parameter uncertainties in the arm dynamics. External forces are applied along the $x$ and $y$ directions at time $t=4[\mathrm{~s}]$ and $t=15[\mathrm{~s}]$, respectively. From these plots, it is possible to note that as expected on the basis of Property 2, the endeffector position error is mainly affected by the external force, that is considered measurable during this test, and exactly related to the desired workspace stiffness. It is important to note that the joint mechanical stiffness is not significantly affected by the remaining parts of the system, then the independent regulation of the actuation stiffness is achieved. The joint stiffness tracking error is not reported, since it is very limited and not meaningful. In Figure 3, also the joint reference trajectory computed by the inverse kinematic solver is reported (upper left plot): this information is useful for the regulation of the null-space behavior of the system. Finally, also the positioning actuators trajectories are reported.

In Figure 4, the results of the case study no. 2 are reported. During this test, a different set of point-to-point trajectories have been evaluated together with an higher values of the desired workspace stiffness and larger (measurable) external forces. The same limitations on the maximum workspace velocity considered in the previous case are assumed during this test. Also, in this simulation, the external forces are applied along $x$ and $y$ at time $t=4[\mathrm{~s}]$ and $t=15[\mathrm{~s}]$, respectively. As in the previous case, the endeffector position error is determined by the external force and by the desired workspace stiffness, while the commanded joint stiffness is the same of the case no. 1, hence showing the independent control of the workspace and of the joints stiffness.

For the simulation of case no. 3, whose results are reported in Figure 5, the same condition of case no. 2 are considered, but the perfect knowledge of the arm parameters is assumed, and the external force has been considered nonmeasurable: from the comparison with Figure 4, it is possible to note that the proposed virtual force sensor is able to correctly estimate the external force; however, the response of the system in case no. 3 shows larger oscillations during transients with respect to case no. 2 , but the response in static conditions is identical to those obtained considering direct measure of the external force, that is, in case no. 2 .

\section{Conclusions}

In this paper, the problem of output-based regulation of robotic manipulators with variable stiffness actuation has been discussed. A control scheme has been proposed whose main features are the simultaneous and decoupled workspace stiffness-position control with limited error, disturbance compensation and estimation-control of the environment interaction force. Moreover, the case of manipulator redundancy has been explicitly addressed and some nice features and implications related to the control of the both the joint mechanical stiffness and workspace stiffness have been highlighted. The control objective is achieved by means of a multilevel controller that decouples the dynamics of both the positioning and the stiffness actuators from the arm dynamics. This result is obtained by applying a singular perturbation analysis to both the actuators and the arm dynamics, configuring in this way a multilevel control structure that presents fast controllers for the actuators and a slow controller for the arm. Moreover, the proposed controller presents several advantages with respect to previous approaches for this class of devices, as the rigid-robot-like trajectory specification, the reduced complexity and the availability of the system residuals for collision detection/ reaction purposes to cite some of them. The extension of the proposed analysis to an hybrid case, in which both rigid 

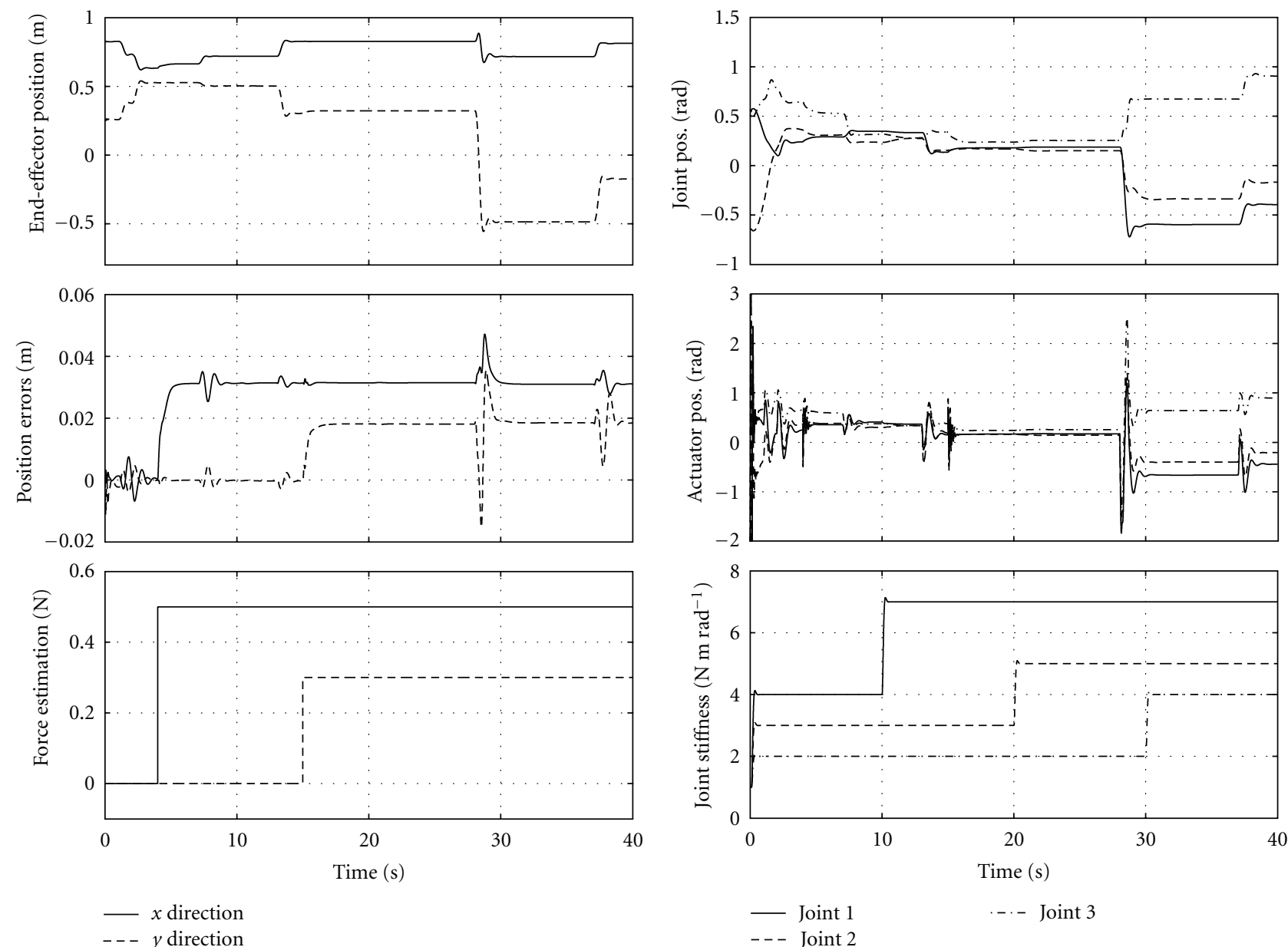

(a)

(b)

FIgURE 3: Response of the system during test no. 1.

and elastic joints are present, with constant or variable stiffness, can be easily achieved. Also, the case of joints with different stiffness variation models can be easily considered. The proposed control approach is validated by means of simulation of a three-link planar manipulator, and the experimental validation of the proposed approach will be considered in the future.

\section{Appendix}

Property 5. The general second-order dynamic system (10) can be used in place of each of (1)-(3) by posing:

(i) In case of (1)

$v=q, \quad w=\left[\begin{array}{ll}\theta & k\end{array}\right], \quad \alpha=\eta_{q}, \quad \tau=\tau_{e}$,

$A(v, w)=M(q), \quad f(\dot{v}, v, w)=N(q, \dot{q})+K[q-\theta]$.

(ii) In case of (2),

$$
\begin{aligned}
& v=\theta, \quad w=[q k], \quad \alpha=\eta_{\theta}, \quad \tau=\tau_{\theta}, \\
& A(v, w)=B, \quad f(\dot{v}, v, w)=K[\theta-q] .
\end{aligned}
$$

(iii) In case of (3),

$$
\begin{aligned}
& v=k, \quad w=[q \theta], \quad \alpha=\eta_{k}, \quad \tau=\tau_{k}, \\
& A(v, w)=\gamma(q, \theta, k), \quad f(\dot{v}, v, w)=\beta(q, \theta, k) .
\end{aligned}
$$

Property 6. The general second-order dynamic system (10) can be used in place of (41)-(43) by posing

$$
\begin{aligned}
& v=q, \quad w=\left[\begin{array}{ll}
\theta & k
\end{array}\right], \quad \alpha=\eta_{q}+\tau_{e}, \quad \tau=\tau_{\mathrm{act}_{d}}, \\
& A(v, w)=M(q), \quad f(\dot{v}, v, w)=N(q, \dot{q}) .
\end{aligned}
$$

Theorem 3. Let $K_{d}, J$, and ${ }^{w} K_{d}$ be $n \times n$ diagonal positive definite, $m \times n$ and $m \times m$ symmetric positive definite matrices, respectively. The following definitions are given:

$$
\begin{gathered}
{ }^{w} K_{j}{ }^{-1}=J K_{d}^{-1} J^{T}, \\
{ }^{w} K_{c}={ }^{w} K_{d}-{ }^{w} K_{j}, \\
K_{a}=K_{d}+J^{T}{ }^{w} K_{c} J,
\end{gathered}
$$



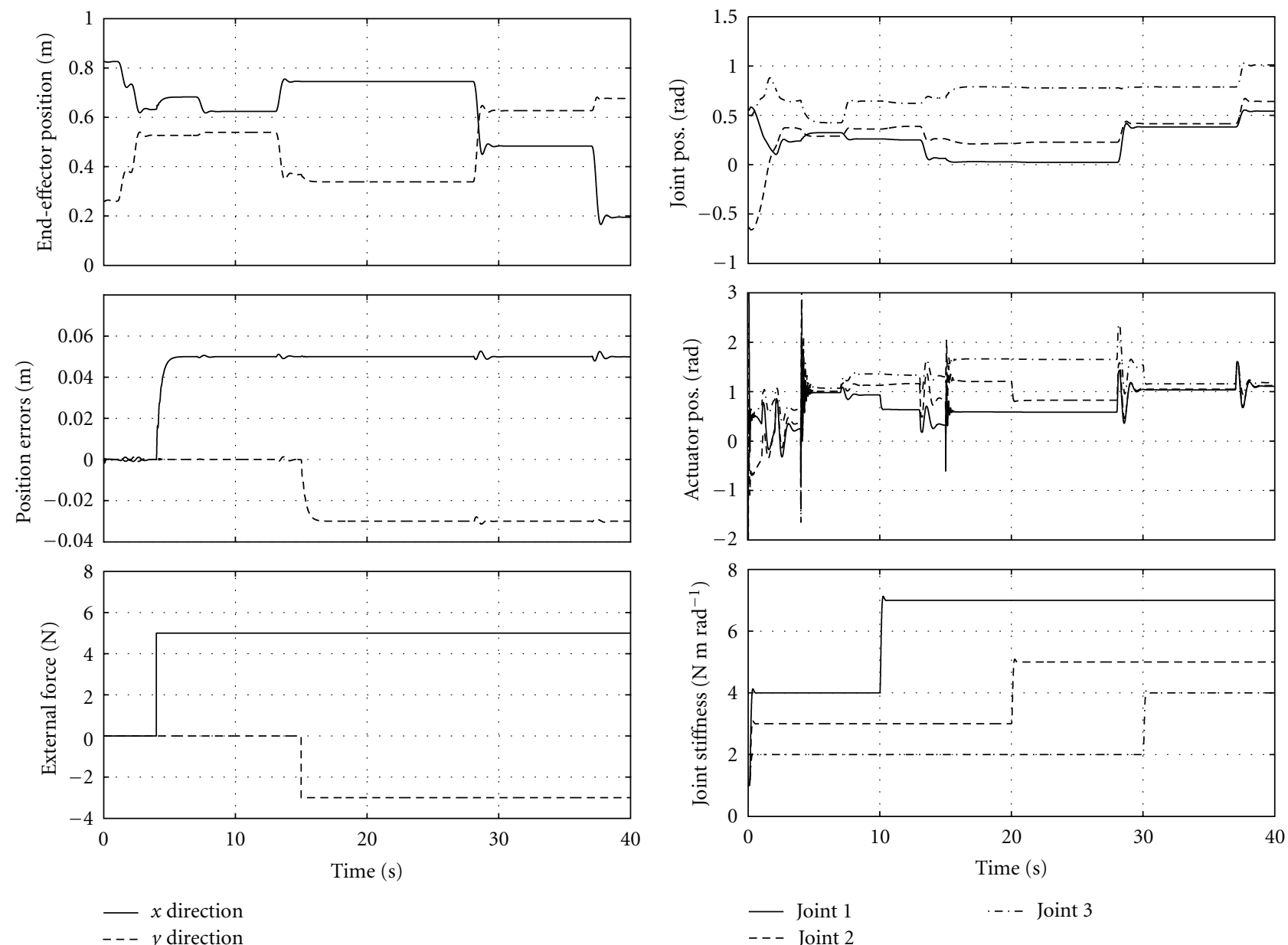

(a)

(b)

FIgURE 4: Response of the system during test no. 2.

where the properties of symmetry and positive definition of the matrices ${ }^{w} K_{j},{ }^{w} K_{c}$, and $K_{a}$ should hold. Note that (A.5)(A.7) correspond to (49)-(51). Then,

$$
\begin{gathered}
J K_{a}^{-1} J^{T}={ }^{w} K_{d}{ }^{-1} \\
K_{a}^{-1} J^{T}\left[J K_{a}^{-1} J^{T}\right]^{-1}=K_{d}^{-1} J^{T}\left[J K_{d}^{-1} J^{T}\right]^{-1} .
\end{gathered}
$$

Proof. Let consider (A.9) first. By applying the matrix inversion lemma [38] to (A.7)

$$
K_{a}^{-1}=K_{d}^{-1}-K_{d}^{-1} J^{T}\left[{ }^{w} K_{c}{ }^{-1}+{ }^{w} K_{j}^{-1}\right]^{-1} J K_{d}^{-1} .
$$

It follows that

$$
J K_{a}^{-1} J^{T}={ }^{w} K_{j}{ }^{-1}-{ }^{w} K_{j}-1\left[{ }^{w} K_{c}{ }^{-1}+{ }^{w} K_{j}{ }^{-1}\right]^{-1}{ }^{w} K_{j}{ }^{-1} .
$$

By applying the matrix inversion lemma also to (A.6), it results

$$
{ }^{w} K_{d}{ }^{-1}={ }^{w} K_{j}^{-1}-{ }^{w} K_{j}-1\left[{ }^{w} K_{c}{ }^{-1}+{ }^{w} K_{j}^{-1}\right]{ }^{-1}{ }^{w} K_{j}^{-1} .
$$

By noticing that (A.11) and (A.12) are equivalent, it follows that the relation (A.8) is satisfied.

Now (A.9) is considered. From (A.10) and (A.8),

$$
\begin{aligned}
& K_{a}^{-1} J^{T}\left[J K_{a}^{-1} J^{T}\right]^{-1} \\
& =\left\{K_{d}^{-1}-K_{d}^{-1} J^{T}\left[{ }^{w} K_{c}{ }^{-1}+{ }^{w} K_{j}-1\right]^{-1} J K_{d}^{-1}\right\} J^{T}{ }^{w} K_{d} .
\end{aligned}
$$

By introducing (A.6)

$$
\begin{aligned}
& K_{a}^{-1} J^{T}\left[J K_{a}^{-1} J^{T}\right]^{-1} \\
& \quad=\left\{K_{d}^{-1}-K_{d}^{-1} J^{T}\left[{ }^{w} K_{c}{ }^{-1}+{ }^{w} K_{j}{ }^{-1}\right]^{-1} J K_{d}^{-1}\right\} \\
& J^{T}\left[{ }^{w} K_{c}+{ }^{w} K_{j}\right]
\end{aligned}
$$



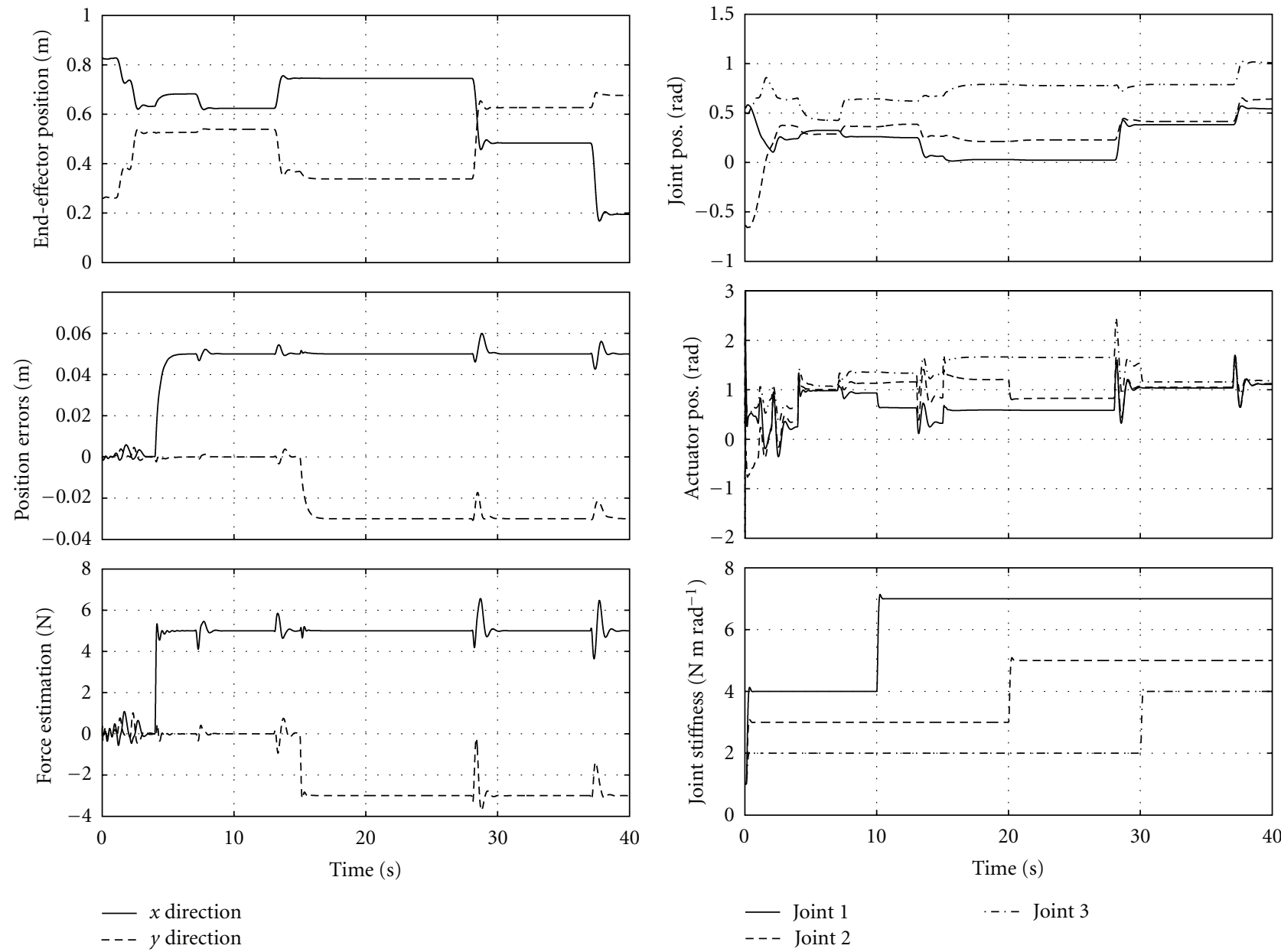

(a)

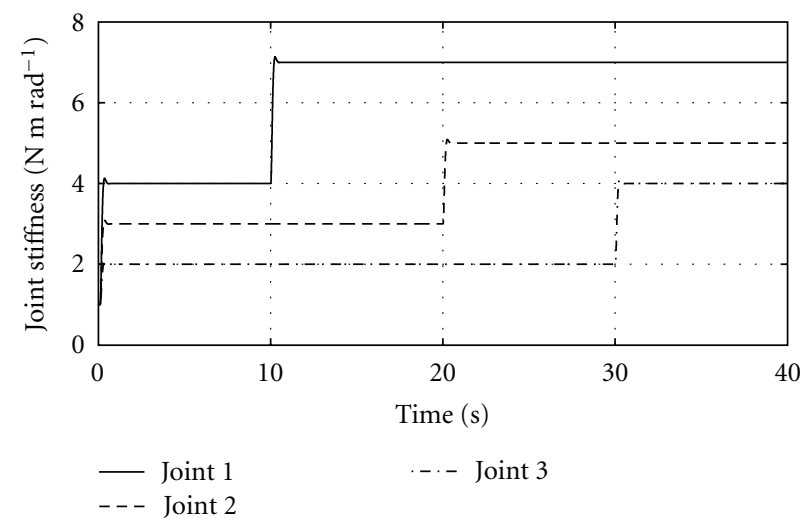

(b)

FIGURE 5: Response of the system during test no. 3.

$$
\begin{aligned}
=K_{d}^{-1} J^{T}\{- & {\left[{ }^{w} K_{c}{ }^{-1}+{ }^{w} K_{j}{ }^{-1}\right]^{-1}{ }^{w} K_{j}{ }^{-1}\left[{ }^{w} K_{c}+{ }^{w} K_{j}\right] } \\
& \left.+{ }^{w} K_{c}+{ }^{w} K_{j}\right\} \\
=K_{d}^{-1} J^{T}\{- & {\left[{ }^{w} K_{c}{ }^{-1}+{ }^{w} K_{j}{ }^{-1}\right]^{-1}\left[{ }^{w} K_{j}{ }^{-1}+{ }^{w} K_{c}{ }^{-1}\right]{ }^{w} K_{c} } \\
& \left.+{ }^{w} K_{c}+{ }^{w} K_{j}\right\}=K_{d}^{-1} J^{T}\left[J K_{d}^{-1} J^{T}\right]^{-1},
\end{aligned}
$$

where (A.5) has been finally used.

\section{Acknowledgments}

This research has been partially supported by the EC Seventh Framework Programme (FP7) under Grant agreement no. 216239 as part of the IP DEXMART (DEXterous and autonomous dual-arm/hand robotic manipulation with sMART sensory-motor skills: a bridge from natural to artificial cognition) and by the Italian MIUR with the PRIN2007CCRNFA-004/PRIN2008-ZRLPWS projects.

\section{References}

[1] C. E. English and D. Russell, "Mechanics and stiffness limitations of a variable stiffness actuator for use in prosthetic limbs," Mechanism and Machine Theory, vol. 34, no. 1, pp. 725, 1999.

[2] A. Bicchi and G. Tonietti, "Fast and soft arm tactics: dealing with the safety-performance trade-off in robot arms design and control," IEEE Robotics and Automation Magazine, vol. 11, no. 2, pp. 22-33, 2004.

[3] G. Berselli and G. Vassura, "Differentiated layer design to modify the compliance of soft pads for robotic limbs," in Proceedings of the IEEE International Conference on Robotics and Automation (ICRA '09), pp. 1285-1290, Kobe, Japan, May 2009.

[4] A. Bicchi, G. Tonietti, M. Bavaro, and M. Piccigallo, "Variable stiffness actuators for fast and safe motion control," in Proceedings of the Robotics Research: The Eleventh International 
Symposium (ISRR '05), vol. 15, pp. 527-536, Springer, Italy, 2005.

[5] S. A. Migliore, E. A. Brown, and S. P. DeWeerth, "Novel nonlinear elastic actuators for passively controlling robotic joint compliance," Journal of Mechanical Design, vol. 129, no. 4, pp. 406-412, 2007.

[6] S. Wolf and G. Hirzinger, "A new variable stiffness design: matching requirements of the next robot generation," in Proceedings of the IEEE International Conference on Robotics and Automation (ICRA '08), pp. 1741-1746, Pasadena, Calif, USA, May 2008.

[7] R. Schiavi, G. Grioli, S. Sen, and A. Bicchi, "VSA-II: a novel prototype of variable stiffness actuator for safe and performing robots interacting with humans," in Proceedings of the IEEE International Conference on Robotics and Automation (ICRA '08), pp. 2171-2176, Pasadena, Calif, USA, 2008.

[8] D. Hyun, H. S. Yang, J. Park, and Y. Shim, "Variable stiffness mechanism for human-friendly robots," Mechanism and Machine Theory, vol. 45, no. 6, pp. 880-897, 2010.

[9] G. Palli, C. Melchiorri, T. Wimböck, M. Grebenstein, and G. Hirzinger, "Feedback linearization and simultaneous stiffnessposition control of robots with antagonistic actuated joints," in Proceedings of the IEEE International Conference on Robotics and Automation (ICRA '07), pp. 4367-4372, Rome, Italy, April 2007.

[10] G. Palli, C. Melchiorri, and A. de Luca, "On the feedback linearization of robots with variable joint stiffness," in Proceedings of the IEEE International Conference on Robotics and Automation (ICRA '08), pp. 1753-1759, Pasadena, Calif, USA, 2008.

[11] G. Palli and C. Melchiorri, "Robust control of robots with variable joint stiffness," in Proceedings of the International Conference on Advanced Robotics (ICAR '09), pp. 1-6, Munich, Germany, June 2009.

[12] A. Bicchi, G. Tonietti, M. Bavaro, and M. Piccigallo, "Variable stiffness actuators for fast and safe motion control," Springer Tracts in Advanced Robotics, vol. 15, pp. 527-536, 2005.

[13] M. W. Spong, "Modeling and control of elastic joint robots," Journal of Dynamic Systems, Measurement and Control, vol. 109, no. 4, pp. 310-319, 1987.

[14] J. K. Salisbury, "Active stiffness control of a manipulator in cartesian coordinates," in Proceedings of the 19th IEEE Conference on Decision and Control including the Symposium on Adaptive Processes, vol. 19, pp. 95-100, December 1980.

[15] N. Hogan, "Impedance control: an approach to manipulation, parts I-III," ASME-Journal of Dynamic Systems, Measurement, and Control, vol. 107, pp. 1-24, 1985.

[16] A. Albu-Schäffer, C. Ott, and G. Hirzinger, "A unified passivity-based control framework for position, torque and impedance control of flexible joint robots," International Journal of Robotics Research, vol. 26, no. 1, pp. 23-39, 2007.

[17] S. Haddadin, A. Albu-Schffer, and G. Hirzinger, "Safety analysis for a human-friendly manipulator," International Journal of Social Robotics, vol. 2, no. 3, pp. 235-252, 2010.

[18] D. W. Robinson, J. E. Pratt, D. J. Paluska, and G. A. Pratt, "Series elastic actuator development for a biomimetic walking robot," in Proceedings of the IEEE/ASME International Conference on Advanced Intelligent Mechatronics (AIM '99), pp. 561-568, Atlanta, Ga, USA, September 1999.

[19] B. T. Knox and J. P. Schmiedeler, "A unidirectional serieselastic actuator design using a spiral torsion spring," Journal of Mechanical Design, vol. 131, no. 12, pp. 125001-125010, 2009.

[20] K. Yokoi, H. Maekawa, and K. Tanie, "A method of compliance control for a redundant manipulator," in Proceedings of the
IEEE/RSJ International Conference on Intelligent Robots and Systems, vol. 3, pp. 1927-1934, July 1992.

[21] T. Wimbock, C. Ott, A. Albu-Schaffer, A. Kugi, and G. Hirzinger, "Impedance control for variable stiffness mechanisms with nonlinear joint coupling," in Proceedings of the IEEE/RSJ International Conference on Intelligent Robots and Systems, pp. 3796-3803, Nice, France, Septemper 2008.

[22] A. Teel and L. Praly, "Global stabilizability and observability imply semi-global stabilizability by output feedback," Systems and Control Letters, vol. 22, no. 5, pp. 313-325, 1994.

[23] B. Yang and W. Lin, "On semi-global stabilizability of MIMO nonlinear systems by output feedback," Automatica, vol. 42, no. 6, pp. 1049-1054, 2006.

[24] A. Isidori, Nonlinear Control Systems II, Springer, Berlin, Germany, 1999.

[25] A. Tornambè, "Output feedback stabilization of a class of non-minimum phase nonlinear systems," Systems and Control Letters, vol. 19, no. 3, pp. 193-204, 1992.

[26] H. K. Khalil, Nonlinear Systems, Prentice Hall, New York, NY, USA, 3rd edition, 2002.

[27] L. Sciavicco and B. Siciliano, Modeling and Control of Robot Manipulators, McGraw-Hill, New York, NY, USA, 1996.

[28] R. V. Patel and F. Shadpey, Control of Redundant Robot Manipulators: Theory and Experiments, Springer, New York, NY, USA, 2009.

[29] H. Y. Choi, W. K. Chung, and Y. Youm, "Stiffness analysis and control of redundant manipulators," in Proceedings of the IEEE International Conference on Robotics and Automation (ICRA '94), vol. 1, pp. 689-695, San Diego, Calif, USA, May 1994.

[30] M. M. Svinin, S. Hosoe, M. Uchiyama, and Z. Luo, "On the stiffness and stiffness control of redundant manipulators," in Proceedings of the IEEE International Conference on Robotics and Automation (ICRA '02), pp. 2393-2399, Washington, DC, USA, May 2002.

[31] W. Yu and X. Li, "PD control of robot with velocity estimation and uncertainties compensation," International Journal of Robotics and Automation, vol. 21, no. 1, pp. 1-9, 2006.

[32] E. Shin and K. Lee, "Robust output feedback control of robot manipulators using high-gain observer," in Proceedings of the IEEE Conference on Control Applications, vol. 1, pp. 881-886, Kohala Coast, Hawaii, USA, August 1999.

[33] P. Tomei, "Robust adaptive friction compensation for tracking control of robots," in Proceedings of the IEEE Conference on Control Applications, vol. 1, pp. 875-880, Kohala Coast, Hawaii, USA, August 1999.

[34] R. Kelly, V. Santibánez, and A. Loría, Control of Robot Manipulators in Joint Space, Springer, New York, NY, USA, 1st edition, 2005.

[35] B. Siciliano and O. Khatib, Eds., Springer Handbook of Robotics, Springer, New York, NY, USA, 2008.

[36] A. de Luca and R. Mattone, "Sensorless robot collision detection and hybrid force/motion control," in Proceedings of the IEEE International Conference on Robotics and Automation (ICRA '05), pp. 999-1004, Barcelona, Spain, April 2005.

[37] A. de Luca, F. Flacco, R. Schiavi, and A. Bicchi, "Nonlinear decoupled motion-stiffness control and collision detection/ reaction for the VSAII variable stiffness device," in Proceedings of the IEEE/RSJ International Conference on Intelligent Robots and Systems, pp. 5487-5494, Saint-Louis, Mo, USA, October 2009.

[38] T. Anderson, An Introduction to Multivariate Statistical Analysis, Wiley-Interscience, New York, NY, USA, 2003. 

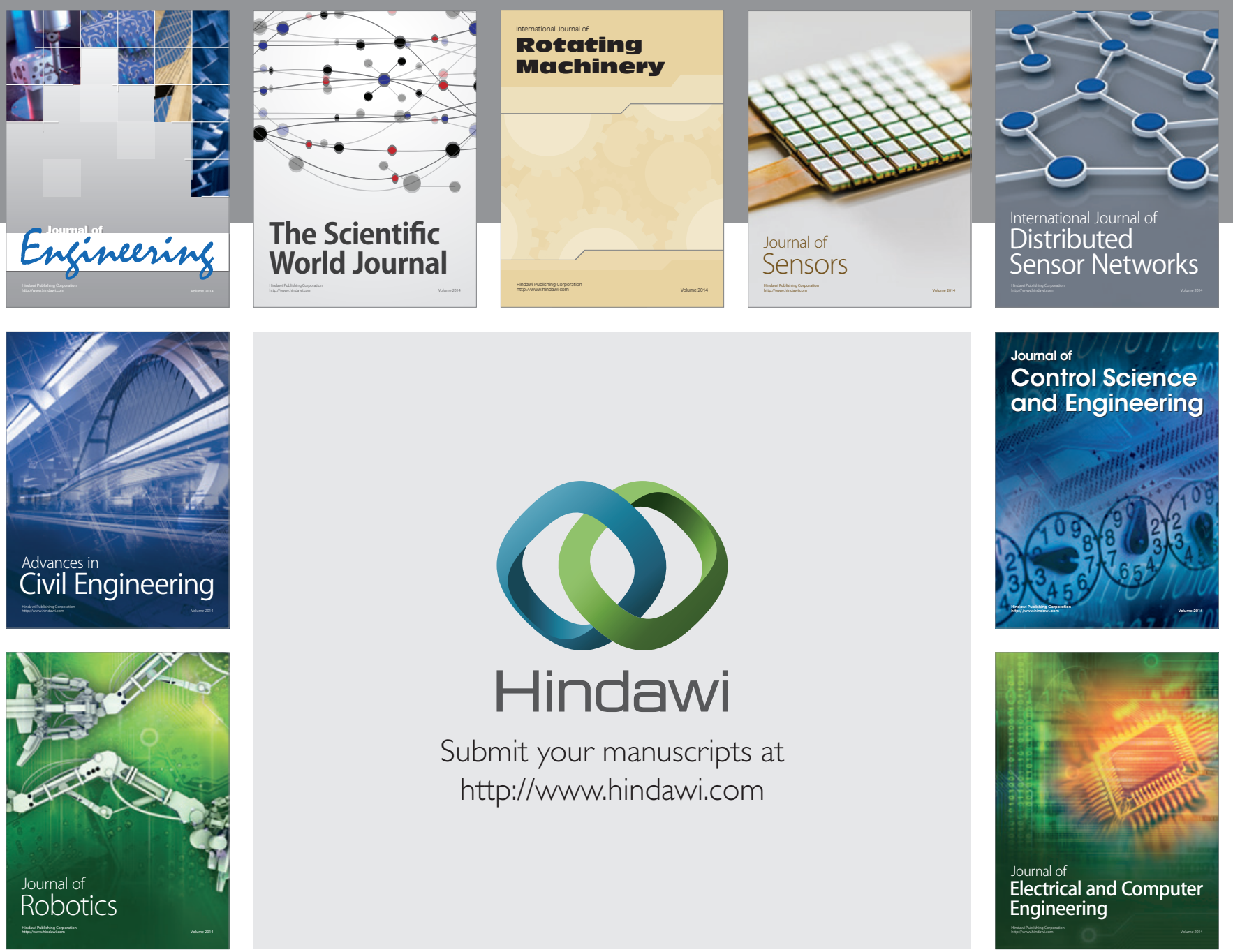

Submit your manuscripts at

http://www.hindawi.com
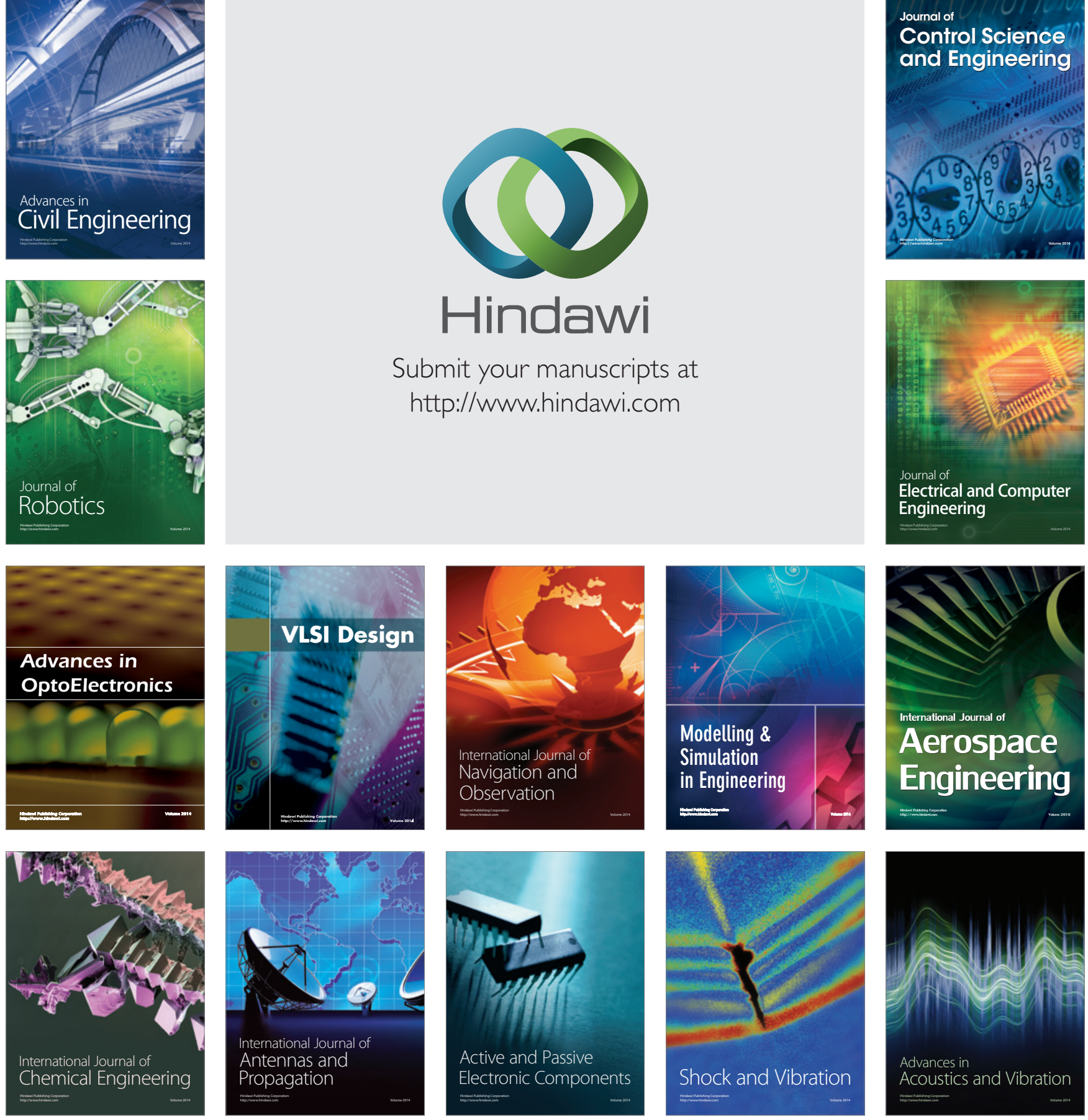